\title{
On the Limit Cycles of a Class of Generalized Kukles Polynomial Differential Systems via Averaging Theory
}

\author{
Amar Makhlouf and Amor Menaceur \\ Department of Mathematics, Faculty of Sciences, University of Annaba, BP 12, Sidi Amar, 23000 Annaba, Algeria \\ Correspondence should be addressed to Amor Menaceur; menaceuramor@yahoo.fr \\ Received 7 October 2014; Accepted 16 December 2014 \\ Academic Editor: Xingfu Zou
}

Copyright (C) 2015 A. Makhlouf and A. Menaceur. This is an open access article distributed under the Creative Commons Attribution License, which permits unrestricted use, distribution, and reproduction in any medium, provided the original work is properly cited.

We apply the averaging theory of first and second order to a class of generalized Kukles polynomial differential systems to study the maximum number of limit cycles of these systems.

\section{Introduction}

One of the main topics in the theory of ordinary differential equations is the study of limit cycles: their existence, their number, and their stability. A limit cycle of a differential equation is an isolated periodic orbit in the set of all periodic orbits of the differential equation. The second part of the 16th Hilbert's problem [1] is related to the least upper bound on the number of limit cycles of polynomial vector fields having a fixed degree. This problem and the Riemann conjecture are the only two problems on the list of Hilbert which have not been solved. Here we consider a very particular case of the sixteenth Hilbert problem. We study the upper bound of the generalized Kukles polynomial system

$$
\dot{x}=-y, \quad \dot{y}=Q(x, y) \text {, }
$$

where $Q(x, y)$ is a polynomial with real coefficients of degree $n$.

Kukles [2], in 1944, introduced the differential system

$$
\begin{aligned}
& \dot{x}=-y, \\
& \dot{y}=x+a_{1} x^{2}+a_{2} x y+a_{3} y^{2}+a_{4} x^{3}+a_{5} x^{2} y+a_{6} x y^{2}+a_{7} y^{3},
\end{aligned}
$$

and he gives the necessary and sufficient conditions in order that this system has a center at the origin. This cubic system without the term $y^{3}$ was also studied in [3] and the authors called it reduced. In [4] a description of the bifurcation of its critical period appears, and [5] presents the existence of reduced Kukles systems with five limit cycles. In the paper [6], the author studied the class of reduced Kukles systems under the cubic perturbation

$$
\begin{gathered}
\dot{x}=-y, \\
\dot{y}=x-\frac{a+1}{a} x^{2}+\frac{2-a}{1-a} y^{2}+\frac{1}{a} x^{3}+\varepsilon g(x, y), \\
g(x, y)=b_{01} y+b_{11} x y+b_{02} y^{2}+b_{12} x y^{2}+b_{21} x^{2} y+b_{03} y^{3},
\end{gathered}
$$

where $\varepsilon>0$ is small and $a>2$ is a constant.

In [7] the author proves that some cubic systems of form (1) can have seven limit cycles. In [8], Chavarriga et al. studied the maximum number of small amplitude limit cycles for Kukles systems which can coexist with some invariant algebraic curves. Also they give a family of cubic Kukles systems

$$
\begin{aligned}
\dot{x}= & -y \\
\dot{y}= & x+\lambda y+a b x^{2}-a\left(a^{2}+3 c-b \lambda\right) x y-2 a b y^{2} \\
& -b^{2} c x^{3}-b\left(b^{2}-a^{2} c-2 c^{2}+b c \lambda\right) x^{2} y \\
& -b^{2}\left(-a^{2}-3 c+b \lambda\right) x y^{2}+b^{3} y^{3}
\end{aligned}
$$


with an invariant hyperbola $h(x, y)=1+a b x-b^{2} c x^{2}-b^{3} x y=$ 0 with $b \neq 0$, which coexist with one or two small amplitude limit cycles. In [9] the author studied the maximum number of limit cycles of the generalized polynomial Liénard differential equations by using the first and second averaging method. In [10], Llibre and Mereu studied the maximum number of limit cycles of the Kukles polynomial differential systems

$$
\begin{aligned}
& \dot{x}=y, \\
& \dot{y}=-x-\sum_{k \geq 1} \varepsilon^{k}\left(f_{n}^{k}(x)+g_{m}^{k}(x) y+h_{l}^{k}(x) y^{2}+d_{0}^{k} y^{3}\right),
\end{aligned}
$$

where for every $k$ the polynomials $f_{n}^{k}(x), g_{m}^{k}(x)$, and $h_{l}^{k}(x)$ have degrees $n, m$, and $l$, respectively, $d_{0}^{k} \neq 0$ is a real number, and $\varepsilon$ is a small parameter.

In this work we study the maximum number of limit cycles given by averaging theory of first and second order, which can bifurcate from the periodic orbits of the linear center $\dot{x}=y, \dot{y}=-x$ perturbed inside the following class of generalized Kukles polynomial differential systems:

$$
\begin{aligned}
& \dot{x}=y, \\
& \dot{y}=-x-\sum_{k \geq 1} \varepsilon^{k}\left(f_{n}^{k}(x)+g_{m}^{k}(x) y+h_{l}^{k}(x) y^{2}+g_{m}^{k}(x) y^{3}\right),
\end{aligned}
$$

where for every $k$ the polynomials $f_{n}^{k}(x), g_{m}^{k}(x)$, and $h_{l}^{k}(x)$ have degrees $n, m$, and $l$, respectively, and $\varepsilon$ is a small parameter. We have considered the same polynomial $g_{m}^{k}(x)$ as the coefficient of $y$ and $y^{3}$. With this choice, we can apply the first and second order of the averaging method. If we consider the coefficients of $y^{i}$ for $i=0,1,2,3$ as arbitrary polynomials, it is difficult to apply the second order averaging method, because to pass from the first order to the second order averaging method, we must put the averaged function of the first order $F_{10}(r)$ (see (8)) identically null. In this case the calculations of the averaged function of the second order $F_{20}(r)$ (see (9)) become difficult. If we replace $d_{0}^{k}$ by $h_{l}^{k}(x)$ in the coefficient of $y^{3}$ of the differential systems (5), we can apply the first order averaging method but it is not easy to apply the second averaging method. To apply the second averaging method, we must put $F_{10}(r)=0$ which is equivalent to cancel all coefficients of the polynomial $F_{10}(r)$. The conditions on the coefficient of $F_{10}(r)$ make the calculations of $F_{20}(r)$ difficult. We have used the averaging method for looking for the limit cycles of many classes of Liénard systems. Here we do the same for Kukles differential systems. Comparatively, with the results of the paper [10], we obtained more limit cycles than the results of this paper. More precisely our main result is the following.

Theorem 1. Assume that for $k=1,2$ the polynomials $f_{n}^{k}(x)$, $g_{m}^{k}(x)$, and $h_{l}^{k}(x)$ have degrees $n, m$, and $l$, respectively, with $n, m, l \geq 1$. Then for $|\varepsilon|$ sufficiently small the maximum number of limit cycles of the Kukles polynomial differential systems (6) bifurcating from the periodic orbits of the linear centre $\dot{x}=$ $y, \dot{y}=-x$, using averaging theory

(a) of first order is

(i) no limit cycle for $m=1$,

(ii) $[(m+2) / 2]$ limit cycles for $m \geq 2$,

(b) of second order is $\max \{[n / 2]+[(m+1) / 2],[l / 2]+[(m+$ $3) / 2],[(m+2) / 2]\}$,

where [·] denotes the integer part function.

\section{First and Second Order Averaging Method}

In proof of our main result we use the averaging theory as it is presented in [11]. Consider the differential system

$$
\dot{x}(t)=\varepsilon F_{1}(x, t)+\varepsilon^{2} F_{2}(x, t)+\varepsilon^{3} R(x, t, \varepsilon),
$$

where $F_{1}, F_{2}: D \times \mathbb{R} \rightarrow \mathbb{R}^{n}, R: D \times \mathbb{R} \times\left(-\varepsilon_{f}, \varepsilon_{f}\right) \rightarrow \mathbb{R}^{n}$ are continuous functions, $T$-periodic in the first variable, and $D$ is an open subset of $\mathbb{R}^{n}$. Assume that the following hypotheses (i) and (ii) hold.

(i) $F_{1}(\cdot, t) \in C^{1}(D)$ for all $t \in \mathbb{R}, F_{1}, F_{2}, R$, and $D_{x} F_{1}$ are locally Lipschitz with respect to $x$, and $R$ is differentiable with respect to $\varepsilon$. We define

$$
\begin{aligned}
& F_{10}(z)=\frac{1}{T} \int_{0}^{T} F_{1}(z, s) d s \\
& F_{20}(z)=\frac{1}{T} \int_{0}^{T}\left[D_{z} F_{1}(z, s) \cdot \int_{0}^{s} F_{1}(z, t) d t+F_{2}(z, s)\right] d s .
\end{aligned}
$$

(ii) For $V \subset D$ an open and bounded set and for each $\varepsilon \in\left(-\varepsilon_{f}, \varepsilon_{f}\right) \backslash\{0\}$, there exists $a_{\varepsilon} \in V$ such that $F_{10}\left(a_{\varepsilon}\right)+$ $F_{20}\left(a_{\varepsilon}\right)=0$ and $d_{B}\left(F_{10}+\varepsilon F_{20}, V, a_{\varepsilon}\right) \neq 0$.

Then, for $|\varepsilon|>0$ sufficiently small there exists a Tperiodic solution $\varphi(\cdot, \varepsilon)$ of system $(7)$ such that $\varphi(0, \varepsilon)=a_{\varepsilon}$.

The expression $d_{B}\left(F_{10}+\varepsilon F_{20}, V, a_{\varepsilon}\right) \neq 0$ means that the Brouwer degree of the function $F_{10}+\varepsilon F_{20}: V \rightarrow \mathbb{R}^{n}$ at the fixed point $a_{\varepsilon}$ is not zero. A sufficient condition for the inequality to be true is that the Jacobian of the function $F_{10}+$ $\varepsilon F_{20}$ at $a_{\varepsilon}$ is not zero.

If $F_{10}$ is not identically zero, then the zeros of $F_{10}+\varepsilon F_{20}$ become mainly the zeros of $F_{10}$ for $\varepsilon$ sufficiently small. In this case the previous result provides the averaging theory of first order.

If $F_{10}$ is identically zero and $F_{20}$ is not identically zero, then the zeros of $F_{10}+\varepsilon F_{20}$ are mainly the zeros of $F_{20}$ for $\varepsilon$ sufficiently small. In this case the previous result provides the averaging theory of second order. For more information about the averaging theory see $[12,13]$.

\section{Proof of Theorem 1}

3.1. Proof of Statement (a) of Theorem 1. In order to apply the first order averaging method we write system (6) with $k=$ 1 , in polar coordinates $(r, \theta)$ where $x=r \cos \theta, y=r \sin \theta$, 
$r>0$. If we take $f_{n}^{1}(x)=\sum_{i=0}^{n} a_{i} x^{i}, g_{m}^{1}(x)=\sum_{i=0}^{m} b_{i} x^{i}$, and $h_{l}^{1}(x)=\sum_{i=0}^{l} c_{i} x^{i}$, system (6) can be written as follows:

$$
\begin{aligned}
\dot{r} & =-\varepsilon \sin \theta P(r, \theta), \\
\dot{\theta} & =-1-\frac{\varepsilon}{r} \cos \theta P(r, \theta),
\end{aligned}
$$

where

$$
\begin{aligned}
P(r, \theta)= & \sum_{i=0}^{n} a_{i} r^{i} \cos ^{i} \theta+\sum_{i=0}^{m} b_{i} r^{i+1} \cos ^{i} \theta \sin \theta \\
& +\sum_{i=0}^{m} b_{i} r^{i+3} \cos ^{i} \theta \sin ^{3} \theta+\sum_{i=0}^{l} c_{i} r^{i+2} \cos ^{i} \theta \sin ^{2} \theta .
\end{aligned}
$$

If we take $\theta$ as a new independent variable, system (10) becomes

$$
\frac{d r}{d \theta}=\varepsilon \sin \theta P(r, \theta)+o\left(\varepsilon^{2}\right)=\varepsilon F_{1}(r, \theta)+o\left(\varepsilon^{2}\right) .
$$

By using the notation introduced in Section 2 we have that

$$
F_{10}(r)=\frac{1}{2 \pi} \int_{0}^{2 \pi} \sin \theta P(r, \theta) d \theta .
$$

We know that

$$
\int_{0}^{2 \pi} \cos ^{i} \theta \sin ^{j} \theta d \theta= \begin{cases}0 & \text { if } i \text { odd or } j \text { is odd } \\ I_{i, j} \neq 0 & \text { if } i \text { is even and } j \text { even. }\end{cases}
$$

Let $k$ be a positive integer. We define ev( $(k)$ as the largest even integer less than or equal to $k$, and $\operatorname{od}(k)$ as the largest odd integer less than or equal to $k$.

Hence

$$
F_{10}(r)=\frac{1}{2 \pi} \sum_{\substack{i=0 \\ i \text { even }}}^{\mathrm{ev}(m)} b_{i} I_{i, 2} r^{i+1}+\frac{1}{2 \pi} \sum_{\substack{i=0 \\ i \text { even }}}^{\mathrm{ev}(m)} b_{i} I_{i, 4} r^{i+3} .
$$

We obtain

$$
\begin{aligned}
F_{10}(r)=\frac{r}{2 \pi}[ & b_{0} I_{0,2}+\left(b_{2} I_{2,2}+b_{0} I_{0,4}\right) r^{2}+\left(b_{4} I_{4,2}+b_{2} I_{2,4}\right) r^{4} \\
& +\cdots+\left(b_{\mathrm{ev}(m)} I_{\operatorname{ev}(m), 2}+b_{\mathrm{ev}(m)-2} I_{\mathrm{ev}(m)-2,4}\right) r^{\operatorname{ev}(m)} \\
& \left.+b_{\mathrm{ev}(m)} r^{\operatorname{ev}(m)+2}\right] .
\end{aligned}
$$

For the case $m=1$, we obtain that

$$
\begin{aligned}
F_{10}(r) & =\frac{b_{0} r}{2 \pi}\left(I_{0,2}+I_{0,4} r^{2}\right) \\
& =\frac{b_{0} r}{2}\left(1+\frac{3}{4} r^{2}\right) .
\end{aligned}
$$

There is no positive root for $F_{10}(r)$.

For $m \geq 2$, the polynomial $F_{10}(r)$ has at most $[(m+2) / 2]$ positive roots. Hence (a) of Theorem 1 is proved.
3.2. Proof of Statement (b) of Theorem 1. For proving statement (b) of Theorem 1 we will use the second-order averaging theory. If we write

$$
\begin{array}{rlrl}
f_{n}^{1}(x) & =\sum_{i=0}^{n} a_{i} x^{i}, & f_{n}^{2}(x)=\sum_{i=0}^{n} \widetilde{a}_{i} x^{i}, \\
g_{m}^{1}(x)=\sum_{i=0}^{m} b_{i} x^{i}, & g_{m}^{2}(x)=\sum_{i=0}^{m} \widetilde{b}_{i} x^{i}, \\
h_{l}^{1}(x)=\sum_{i=0}^{l} c_{i} x^{i}, & h_{l}^{2}(x)=\sum_{i=0}^{l} \widetilde{c}_{i} x^{i}
\end{array}
$$

then system (6) with $k=2$ in polar coordinates $(r, \theta), r>0$, becomes

$$
\begin{aligned}
& \dot{r}=-\varepsilon \sin \theta P(r, \theta)-\varepsilon^{2} \sin \theta K(r, \theta), \\
& \dot{\theta}=-1-\frac{\varepsilon}{r} \cos \theta P(r, \theta)-\frac{\varepsilon^{2}}{r} \cos \theta K(r, \theta),
\end{aligned}
$$

where

$$
\begin{aligned}
K(r, \theta)= & \sum_{i=0}^{n} \widetilde{a}_{i} r^{i} \cos ^{i} \theta+\sum_{i=0}^{m} \widetilde{b}_{i} r^{i+1} \cos ^{i} \theta \sin \theta \\
& +\sum_{i=0}^{m} \widetilde{b}_{i} r^{i+3} \cos ^{i} \theta \sin ^{3} \theta+\sum_{i=0}^{l} \widetilde{c}_{i} r^{i+2} \cos ^{i} \theta \sin ^{2} \theta .
\end{aligned}
$$

Taking $\theta$ as the new independent variable system, (19) can be written as

$$
\frac{d r}{d \theta}=\varepsilon F_{1}(r, \theta)+\varepsilon^{2} F_{2}(r, \theta)+o\left(\varepsilon^{3}\right),
$$

where $F_{1}(r, \theta)=\sin \theta P(r, \theta)$ and $F_{2}(r, \theta)=\sin \theta K(r, \theta)-$ $(\cos \theta \sin \theta / r) P^{2}(r, \theta)$.

In order to apply the averaging theory of second order, $F_{10}$ must be identically zero. Therefore from (16), $F_{10}$ is identically zero if and only if $b_{i}=0$ for $i$ even.

Now we determine the corresponding function

$$
F_{20}=\frac{1}{2 \pi} \int_{0}^{2 \pi}\left[\frac{d}{d r} F_{1}(r, \theta) \cdot \int_{0}^{\theta} F_{1}(r, \phi) d \phi+F_{2}(r, \theta)\right] d \theta .
$$

For this we compute

$$
\begin{aligned}
& \frac{d}{d r} F_{1}(r, \theta) \\
& =\sum_{i=1}^{n} i a_{i} r^{i-1} \cos ^{i} \theta \sin \theta \\
& \quad+\sum_{\substack{i=1 \\
i \text { odd }}}^{\text {od }(m)}(i+1) b_{i} r^{i} \cos ^{i} \theta \sin ^{2} \theta
\end{aligned}
$$




$$
\begin{aligned}
& +\sum_{\substack{i=1 \\
i \text { odd }}}^{\text {od }(m)}(i+3) b_{i} r^{i+2} \cos ^{i} \theta \sin ^{4} \theta \\
& +\sum_{i=0}^{l}(i+2) c_{i} r^{i+1} \cos ^{i} \theta \sin ^{3} \theta, \\
& \int_{0}^{\theta} F_{1}(r, \phi) d \phi \\
& =\sum_{j=0}^{n} a_{j} r^{j} \int_{0}^{\theta} \cos ^{j} \phi \sin \phi d \phi \\
& +\sum_{\substack{j=1 \\
j \text { odd }}}^{\mathrm{od}(m)} b_{j} r^{j+1} \int_{0}^{\theta} \cos ^{j} \phi \sin ^{2} \phi d \phi \\
& +\sum_{\substack{j=1 \\
j \text { odd }}}^{\text {od }(m)} b_{j} r^{j+3} \int_{0}^{\theta} \cos ^{j} \phi \sin ^{4} \phi d \phi \\
& +\sum_{j=0}^{l} c_{j} r^{j+2} \int_{0}^{\theta} \cos ^{j} \phi \sin ^{3} \phi d \phi \\
& =\sum_{j=0}^{n} a_{j} r^{j} \frac{1}{j+1}\left(1-\cos ^{j+1} \theta\right) \\
& +\sum_{\substack{j=1 \\
j \text { odd }}}^{\text {od }(m)} b_{j} r^{j+1}\left(\sum_{k=1}^{(j+3) / 2} \alpha_{k, j} \sin ((2 k-1) \theta)\right) \\
& +\sum_{\substack{j=1 \\
j \text { odd }}}^{\text {od }(m)} b_{j} r^{j+3}\left(\sum_{k=1}^{(j+5) / 2} \beta_{k, j} \sin ((2 k-1) \theta)\right) \\
& +\sum_{\substack{j=0 \\
j \text { even }}}^{\text {ev }(l)} c_{j} r^{j+2}\left(\gamma_{1, j}+\sum_{k=2}^{(j+6) / 2} \gamma_{k, j} \cos ((2 k-3) \theta)\right) \\
& +\sum_{\substack{j=1 \\
j \text { odd }}}^{\mathrm{od}(l)} c_{j} r^{j+2}\left(\delta_{1, j}+\sum_{k=2}^{(j+5) / 2} \delta_{k, j} \cos ((2 k-2) \theta)\right),
\end{aligned}
$$

where $\alpha_{k, j}, \beta_{k, j}, \gamma_{k, j}$, and $\delta_{k, j}$ are constants.

The integral $\int_{0}^{2 \pi}(d / d r) F_{1}(r, \theta)\left(\int_{0}^{\theta} F_{1}(r, \phi) d \phi\right) d \theta$ will be given in several lemmas.

\section{Lemma 2. The integral}

$$
\int_{0}^{2 \pi}\left(\sum_{i=1}^{n} i a_{i} r^{i-1} \cos ^{i} \theta \sin \theta\right)\left(\int_{0}^{\theta} F_{1}(r, \phi) d \phi\right) d \theta
$$

$i s$, in the variable $r$, the polynomial

$$
\sum_{\substack{i=2 \\ \text { ieven }}}^{e v(n)} \sum_{\substack{j=1 \\ \text { jodd }}}^{\text {od }(m)} i a_{i} b_{j} r^{i+j} A_{i j}+\sum_{\substack{i=2 \\ i \text { even }}}^{e v(n)} \sum_{\substack{j=1 \\ \text { jodd }}}^{\text {od }(m)} i a_{i} b_{j} r^{i+j+2} B_{i j},
$$

where $A_{i j}$ and $B_{i j}$ are real constants.

Proof. We have that

$\left(a_{1}\right)$

$$
\begin{aligned}
\int_{0}^{2 \pi}\left(\sum_{i=1}^{n} i a_{i} r^{i-1} \cos ^{i} \theta \sin \theta\right) \\
\cdot\left(\sum_{j=0}^{n} a_{j} r^{j} \frac{1}{j+1}\left(1-\cos ^{j+1} \theta\right)\right) d \theta=0,
\end{aligned}
$$

$\left(b_{1}\right)$

$$
\begin{aligned}
& \int_{0}^{2 \pi}\left(\sum_{i=1}^{n} i a_{i} r^{i-1} \cos ^{i} \theta \sin \theta\right)
\end{aligned}
$$

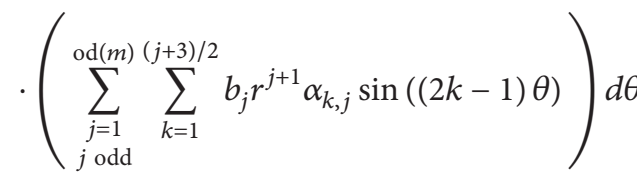

$$
=\sum_{\substack{i=2 \\ i \text { even }}}^{\operatorname{ev}(n)} \sum_{j=1}^{\mathrm{od}(m)} i a_{i} b_{j} r^{i+j} A_{i j}
$$

where $A_{i j}=\int_{0}^{2 \pi} \cos ^{i} \theta \sin \theta\left(\sum_{k=1}^{(j+3) / 2} \alpha_{k, j} \sin ((2 k-\right.$ 1) $\theta)) d \theta \neq 0$ for $i \geqslant 2$ even and $j \geqslant 1$ odd,

$\left(c_{1}\right)$

$$
\begin{aligned}
& \int_{0}^{2 \pi}\left(\sum_{i=1}^{n} i a_{i} r^{i-1} \cos ^{i} \theta \sin \theta\right) \\
& \quad\left(\sum_{\substack{j=0 \\
j \text { even }}}^{\text {ev }(l)} c_{j} r^{j+2}\left(\gamma_{1, j}+\sum_{k=2}^{(j+6) / 2} \gamma_{k, j} \cos ((2 k-3) \theta)\right)\right) d \theta \\
& =0,
\end{aligned}
$$

$\left(\mathrm{d}_{1}\right)$

$\int_{0}^{2 \pi}\left(\sum_{i=1}^{n} i a_{i} r^{i-1} \cos ^{i} \theta \sin \theta\right)$

$$
\cdot\left(\sum_{\substack{j=1 \\ j \text { odd }}}^{\operatorname{od}(l)} c_{j} r^{j+2}\left(\delta_{1, j}+\sum_{k=2}^{(j+5) / 2} \delta_{k, j} \cos ((2 k-2) \theta)\right)\right) d \theta
$$

$=0$, 
$\left(e_{1}\right)$

$$
\begin{aligned}
& \int_{0}^{2 \pi}\left(\sum_{i=1}^{n} i a_{i} r^{i-1} \cos ^{i} \theta \sin \theta\right) \\
& \cdot\left(\sum_{\substack{j=1 \\
j \text { odd }}}^{\text {od }(m)(j+5) / 2} \sum_{k=1}^{(j)} b_{j} r_{k}^{j+3} \beta_{k, j} \sin ((2 k-1) \theta)\right) d \theta \\
& =\sum_{\substack{i=2 \\
i \text { even }}}^{\operatorname{evv}(n) \text { od }(m)} i a_{i} b_{j} r^{i+j+2} B_{i j},
\end{aligned}
$$

where $B_{i j}=\int_{0}^{2 \pi} \cos ^{i} \theta \sin \theta\left(\sum_{k=1}^{(j+5) / 2} \beta_{k, j} \sin ((2 k-\right.$ 1) $\theta$ )d $d \theta) \neq 0$ for $i \geqslant 2$ even and $j \geqslant 1$ odd.

We have that the sum of the integrals $\left(a_{1}\right)-\left(e_{1}\right)$ is polynomial (3.6). This ends the proof of the lemma.

Lemma 3. The integral

$$
\int_{0}^{2 \pi}\left(\sum_{i=0}^{m}(i+1) b_{i} r^{i} \cos ^{i} \theta \sin ^{2} \theta\right)\left(\int_{0}^{\theta} F_{1}(r, \phi) d \phi\right) d \theta
$$

is, in the variable $r$, the polynomial

$$
\begin{aligned}
& -\sum_{\substack{1 \leq i \leq o d(m) \\
0 \leq j \leq e v(n) \\
i \text { odd and jeven }}} \frac{i+1}{j+1} a_{j} b_{i} r^{i+j} I_{i+j+1,2} \\
& +\sum_{\substack{i=1 \\
\text { iodd }}}^{\text {od }(m)} \sum_{\substack{j e v e n \\
\text { jeven }}}^{e v(l)}(i+1) b_{i} c_{j} r^{i+j+2} D_{i j},
\end{aligned}
$$

where $I_{i+j+1,2}$ and $D_{i j}$ are real constants.

Proof. We have that

$\left(\mathrm{a}_{2}\right)$

$$
\begin{array}{r}
\int_{0}^{2 \pi}\left(\sum_{\substack{i=1 \\
i \text { odd }}}^{\operatorname{od}(m)}(i+1) b_{i} r^{i} \cos ^{i} \theta \sin ^{2} \theta\right) \\
\cdot\left(\sum_{j=0}^{n} a_{j} r^{j} \frac{1}{j+1}\left(1-\cos ^{j+1} \theta\right)\right) d \theta \\
=-\sum_{\substack{1 \leq i \leq \mathrm{od}(m) \\
0 \leq j \leq \mathrm{ev}(n) \\
i \text { odd and } j \text { even }}} \frac{i+1}{j+1} a_{j} b_{i} r^{i+j} I_{i+j+1,2},
\end{array}
$$

where $I_{i+j+1,2}=\int_{0}^{2 \pi}\left(\cos ^{i+j+1} \theta \sin ^{2} \theta\right) d \theta \neq 0$ for $i$ odd and $j$ even. $\left(b_{2}\right)$

$$
\begin{aligned}
\int_{0}^{2 \pi}\left(\sum_{\substack{i=1 \\
i \text { odd }}}^{\operatorname{od}(m)}(i+1) b_{i} r^{i} \cos ^{i} \theta \sin ^{2} \theta\right) \\
\quad\left(\sum_{\substack{j=1 \\
j \text { odd }}}^{\operatorname{od}(m)} \sum_{k=1}^{(j+3) / 2} b_{j} r^{j+1} \alpha_{k, j} \sin ((2 k-1) \theta)\right) d \theta=0,
\end{aligned}
$$

$\left(c_{2}\right)$

$$
\int_{0}^{2 \pi}\left(\sum_{\substack{i=1 \\ i \text { odd }}}^{\mathrm{od}(m)}(i+1) b_{i} r^{i} \cos ^{i} \theta \sin ^{2} \theta\right)
$$

$$
\begin{aligned}
& \left(\sum_{\substack{j=0 \\
j \text { even }}}^{\operatorname{ev}(l)} c_{j} r^{j+2}\left(\gamma_{1, j}+\sum_{k=2}^{(j+6) / 2} \gamma_{k, j} \cos ((2 k-3) \theta)\right) d \theta\right. \\
= & \sum_{\substack{i=1 \\
i \text { odd }}}^{\operatorname{od}(m)} \sum_{\substack{j=0 \\
j \text { even }}}^{\operatorname{ev}(l)}(i+1) b_{i} c_{j} r^{i+j+2} D_{i j},
\end{aligned}
$$

where $D_{i j}=\int_{0}^{2 \pi} \cos ^{i} \theta \sin ^{2} \theta\left(\gamma_{1, j}+\right.$ $\left.\sum_{k=2}^{(j+6) / 2} \gamma_{k, j} \cos ((2 k-3) \theta)\right) d \theta \neq 0$ for $i \geqslant 1$ odd and $j \geqslant 0$ even,

$\left(\mathrm{d}_{2}\right)$

$$
\begin{aligned}
& \int_{0}^{2 \pi}\left(\sum_{\substack{i=1 \\
i \text { odd }}}^{\mathrm{od}(m)}(i+1) b_{i} r^{i} \cos ^{i} \theta \sin ^{2} \theta\right) \\
& \quad\left(\sum_{\substack{j=1 \\
j \text { odd }}}^{\text {od }(l)} c_{j} r^{j+2}\left(\delta_{1, j}+\sum_{k=2}^{(j+5) / 2} \delta_{k, j} \cos ((2 k-2) \theta)\right)\right) d \theta \\
& =0
\end{aligned}
$$

$\left(e_{2}\right)$

$$
\begin{aligned}
\int_{0}^{2 \pi} & \left(\sum_{\substack{i=1 \\
i \text { odd }}}^{\operatorname{od}(m)}(i+1) b_{i} r^{i} \cos ^{i} \theta \sin ^{2} \theta\right) \\
& \left(\sum_{\substack{j=1 \\
j \text { odd }}}^{\operatorname{od}(m)} \sum_{k=1}^{(j+5) / 2} b_{j} r^{j+3} \beta_{k, j} \sin ((2 k-1) \theta)\right) d \theta=0 .
\end{aligned}
$$


We have that the sum of the integrals $\left(a_{2}\right)-\left(e_{2}\right)$ is polynomial (32). This ends the proof of the lemma.

Lemma 4. The integral

$$
\int_{0}^{2 \pi}\left(\sum_{i=0}^{l}(i+2) c_{i} r^{i+1} \cos ^{i} \theta \sin ^{3} \theta\right)\left(\int_{0}^{\theta} F_{1}(r, \phi) d \phi\right) d \theta
$$

is, in the variable $r$, the polynomial

$$
\begin{aligned}
\sum_{\substack{i=0 \\
\text { ieven }}}^{e v(l)} \sum_{\substack{j=1 \\
j \text { odd }}}^{\text {od }(m)}(i+2) c_{i} b_{j} r^{i+j+2} E_{i j} \\
+\sum_{\substack{i=0 \\
\text { ieven }}}^{\operatorname{ev}(l)} \sum_{\substack{j=1 \\
j \text { odd }}}^{\text {od }(m)}(i+2) c_{i} b_{j} r^{i+j+4} F_{i j},
\end{aligned}
$$

where $E_{i j}$ and $F_{i j}$ are real constants.

Proof. We have that

$\left(a_{3}\right)$

$$
\begin{aligned}
\int_{0}^{2 \pi} & \left(\sum_{i=0}^{l}(i+2) c_{i} r^{i+1} \cos ^{i} \theta \sin ^{3} \theta\right) \\
& \left(\sum_{j=0}^{n} a_{j} r^{j} \frac{1}{J+1}\left(1-\cos ^{j+1} \theta\right)\right) d \theta=0,
\end{aligned}
$$

$\left(\mathrm{b}_{3}\right)$

$$
\begin{aligned}
\int_{0}^{2 \pi} & \left(\sum_{i=0}^{l}(i+2) c_{i} r^{i+1} \cos ^{i} \theta \sin ^{3} \theta\right) \\
& \cdot\left(\sum_{j=1}^{\operatorname{od}(m)} \sum_{k=1}^{(j+3) / 2} b_{j} r^{j+1} \alpha_{k, j} \sin ((2 k-1) \theta)\right) d \theta \\
= & \sum_{\substack{i=0 \\
i \text { odd }}}^{\operatorname{ev}(l)} \sum_{j=1}^{\text {od }(m)}(i+2) c_{i} b_{j} r^{i+j+2} E_{i j},
\end{aligned}
$$

where $E_{i j}=\int_{0}^{2 \pi} \cos ^{i} \theta \sin ^{3} \theta\left(\sum_{k=1}^{(j+3) / 2} \alpha_{k, j} \sin ((2 k-\right.$ 1) $\theta)) \neq 0$ for $i \geqslant 0$ even and $j \geqslant 1$ odd, $\left(c_{3}\right)$

$$
\begin{aligned}
\int_{0}^{2 \pi} & \left(\sum_{i=0}^{l}(i+2) c_{i} r^{i+1} \cos ^{i} \theta \sin ^{3} \theta\right) \\
& \left(\sum_{\substack{j=0 \\
j \text { even }}}^{\operatorname{ev}(l)} c_{j} r^{j+2}\left(\gamma_{1, j}+\sum_{k=2}^{(j+6) / 2} \gamma_{k, j} \cos ((2 k-3) \theta)\right)\right) d \theta
\end{aligned}
$$$$
=0 \text {, }
$$

$\left(d_{3}\right)$

$$
\begin{aligned}
& \int_{0}^{2 \pi}\left(\sum_{i=0}^{l}(i+2) c_{i} r^{i+1} \cos ^{i} \theta \sin ^{3} \theta\right) \\
& \quad\left(\sum_{\substack{j=1 \\
j \text { odd }}}^{\operatorname{od}(l)} c_{j} r^{j+2}\left(\delta_{1, j}+\sum_{k=2}^{(j+5) / 2} \delta_{k, j} \cos ((2 k-2) \theta)\right)\right) d \theta \\
& =0,
\end{aligned}
$$

$$
\begin{aligned}
& \int_{0}^{2 \pi}\left(\sum_{i=0}^{l}(i+2) c_{i} r^{i+1} \cos ^{i} \theta \sin ^{3} \theta\right) \\
& \quad\left(\sum_{j=1}^{\operatorname{od}(m)} \sum_{k=1}^{(j+5) / 2} b_{j} r^{j+3} \beta_{k}^{j} \sin ((2 k-1) \theta)\right) d \theta \\
& =\sum_{\substack{i=0 \\
i \text { odd }}}^{\operatorname{ev}(l)} \sum_{j=1}^{\text {od }(m)}(i+2) c_{i} b_{j} r^{i+j+4} F_{i j},
\end{aligned}
$$

where $F_{i j}=\int_{0}^{2 \pi} \cos ^{i} \theta \sin ^{3} \theta\left(\sum_{k=1}^{(j+5) / 2} \beta_{k, j} \sin ((2 k-\right.$ 1) $\theta)) \neq 0$ for $i \geqslant 0$ even and $j \geqslant 1$ odd.

We have that the sum of the integrals $\left(a_{3}\right)-\left(e_{3}\right)$ is polynomial (39). This completes the proof of the lemma.

Lemma 5. The integral

$$
\int_{0}^{2 \pi}\left(\sum_{\substack{i=1 \\ i \text { odd }}}^{o d(m)}(i+3) b_{i} r^{i+2} \cos ^{i} \theta \sin ^{4} \theta\right)\left(\int_{0}^{\theta} F_{1}(r, \phi) d \phi\right) d \theta
$$

is, in the variable $r$, the polynomial

$$
\begin{gathered}
-\sum_{\substack{1 \leq i \leq o d(m) \\
0 \leq j \leq e v(n) \\
\text { odd and jeven }}} \frac{i+3}{j+1} a_{j} b_{i} r^{i+j+2} I_{i+j+1,4} \\
+\sum_{\substack{i=1 \\
\text { iodd }}} \sum_{\substack{j=0 \\
\text { jeven }}}^{\text {od }(m)}(i+1) b_{i} c_{j} r^{i+j+4} H_{i j},
\end{gathered}
$$

where $I_{i+j+1,4}$ and $H_{i j}$ are real constants. 
Proof. We have that

$\left(a_{4}\right)$

$$
\begin{gathered}
\int_{0}^{2 \pi}\left(\sum_{\substack{i=1 \\
i \text { odd }}}^{\operatorname{od}(m)}(i+3) b_{i} r^{i+2} \cos ^{i} \theta \sin ^{4} \theta\right) \\
\cdot\left(\sum_{j=0}^{n} a_{j} r^{j} \frac{1}{J+1}\left(1-\cos ^{j+1} \theta\right)\right) d \theta \\
=-\sum_{\substack{1 \leq i \leq \text { od }(m) \\
0 \leq j \leq \operatorname{ev}(n) \\
i \text { odd and } j \text { even }}} \frac{i+3}{j+1} a_{j} b_{i} r^{i+j+2} I_{i+j+1,4},
\end{gathered}
$$

where $I_{i+j+1,4}=\int_{0}^{2 \pi}\left(\cos ^{i+j+1} \theta \sin ^{4} \theta\right) d \theta \neq 0$ for $i$ odd and $j$ even,

$\left(b_{4}\right)$

$$
\begin{aligned}
\int_{0}^{2 \pi}\left(\sum_{\substack{i=1 \\
i \text { odd }}}^{\operatorname{od}(m)}(i+3) b_{i} r^{i+2} \cos ^{i} \theta \sin ^{4} \theta\right) \\
\\
\left.\qquad \sum_{\substack{j=1 \\
j \text { odd }}}^{\operatorname{od}(m)} \sum_{k=1}^{(j+3) / 2} b_{j} r^{j+1} \alpha_{k, j} \sin ((2 k-1) \theta)\right) d \theta=0,
\end{aligned}
$$

$\left(c_{4}\right)$

$$
\begin{aligned}
& \int_{0}^{2 \pi}\left(\sum_{\substack{i=1 \\
i \text { odd }}}^{\operatorname{od}(m)}(i+3) b_{i} r^{i+2} \cos ^{i} \theta \sin ^{4} \theta\right) \\
& \quad\left(\sum_{\substack{j=0 \\
j \text { even }}}^{\operatorname{ev}(l)} c_{j} r^{j+2}\left(\gamma_{1, j}+\sum_{k=2}^{(j+6) / 2} \gamma_{k, j} \cos ((2 k-3) \theta)\right)\right) d \theta \\
& =\sum_{\substack{i=1 \\
i \text { odd }}}^{\substack{\text { od }(m) \\
j \text { even }}}(i+1) b_{i} c_{j} r^{i+j+4} H_{i j},
\end{aligned}
$$

where $H_{i j}=\int_{0}^{2 \pi} \cos ^{i} \theta \sin ^{4} \theta\left(\gamma_{1, j}+\right.$ $\left.\sum_{k=2}^{(j+6) / 2} \gamma_{k, j} \cos ((2 k-3) \theta)\right) d \theta \neq 0$ for $i \geqslant 1$ odd and $j \geqslant 0$ even, $\left(\mathrm{d}_{4}\right)$

$$
\begin{aligned}
& \int_{0}^{2 \pi}\left(\sum_{\substack{i=1 \\
i \text { odd }}}^{\operatorname{od}(m)}(i+3) b_{i} r^{i+2} \cos ^{i} \theta \sin ^{4} \theta\right) \\
& \cdot\left(\sum_{\substack{j=1 \\
j \text { odd }}}^{\operatorname{od}(l)} c_{j} r^{j+2}\left(\delta_{1, j}+\sum_{k=2}^{(j+5) / 2} \delta_{k, j} \cos ((2 k-2) \theta)\right)\right) d \theta \\
& =0 \text {, } \\
& \left(\mathrm{e}_{4}\right) \\
& \int_{0}^{2 \pi}\left(\sum_{\substack{i=1 \\
i \text { odd }}}^{\operatorname{od}(m)}(i+3) b_{i} r^{i+2} \cos ^{i} \theta \sin ^{4} \theta\right) \\
& \cdot\left(\sum_{\substack{j=1 \\
j \text { odd }}}^{\operatorname{od}(m)} \sum_{k=1}^{(j+3) / 2} b_{j} r^{j+3} \beta_{k, j} \sin ((2 k-1) \theta)\right) d \theta=0 .
\end{aligned}
$$

We have that the sum of the integrals $\left(a_{4}\right)-\left(e_{4}\right)$ is polynomial (46). This ends the proof of the lemma.

From Lemmas 2-5 we have that

$$
\begin{aligned}
& \int_{0}^{2 \pi} \frac{d}{d r} F_{1}(r, \theta)\left(\int_{0}^{\theta} F_{1}(r, \phi) d \phi\right) d \theta \\
& =\sum_{\substack{i=2 \\
i \text { even }}}^{\operatorname{ev}(n)} \sum_{\substack{j=1 \\
j \text { odd }}}^{\operatorname{od}(m)} i a_{i} b_{j} r^{i+j} A_{i j}+\sum_{\substack{i=2 \\
i \text { even }}}^{\operatorname{ev}(n)} \sum_{j=1}^{\operatorname{od}(m)} i a_{i} b_{j} r^{i+j+2} B_{i j} \\
& -\sum_{\substack{1 \leq i \leq \mathrm{od}(m) \\
0 \leq j \leq \mathrm{ev}(n)}} \frac{i+1}{j+1} a_{j} b_{i} r^{i+j} I_{i+j+1,2} \\
& +\sum_{\substack{i=1 \\
i \text { odd } \operatorname{\text {odd}} j \text { even }}}^{\substack{\text { od }(m) \\
j \text { even }}}(i+1) b_{i} c_{j} r^{i+j+2} D_{i j} \\
& +\sum_{\substack{i=0 \\
i \text { even }}}^{\substack{\text { ev }(l) \\
j \text { od odd }}}(i+2) c_{i} b_{j} r^{i+j+2} E_{i j}
\end{aligned}
$$$$
+\sum_{\substack{i=0 \\ i \text { even }}}^{\operatorname{ev}(l)} \sum_{\substack{j=1 \\ j \text { odd }}}^{\mathrm{od}(m)}(i+2) c_{i} b_{j} r^{i+j+4} F_{i j}
$$ 


$$
\begin{aligned}
& -\sum_{\substack{1 \leq i \leq \mathrm{od}(m) \\
0 \leq j \leq \mathrm{ev}(n) \\
i \text { odd and } j \text { even }}} \frac{i+3}{j+1} a_{j} b_{i} r^{i+j+2} I_{i+j+1,4} \\
& +\sum_{\substack{i=1 \\
i \text { odd }}} \sum_{\substack{j=0 \\
j \text { even }}}^{\operatorname{od}(m)}(i+1) b_{i} c_{j} r^{i+j+4} H_{i j} .
\end{aligned}
$$

Now we calculate the integral $\int_{0}^{2 \pi} F_{2}(r, \theta) d \theta$.

$$
\begin{aligned}
\int_{0}^{2 \pi} F_{2}(r, \theta) d \theta= & \int_{0}^{2 \pi} \sin \theta K(r, \theta) d \theta \\
& -\int_{0}^{2 \pi} \frac{\sin \theta \cos \theta}{r} P^{2}(r, \theta) d \theta .
\end{aligned}
$$

First we calculate $\int_{0}^{2 \pi} \sin \theta K(r, \theta) d \theta$. Noting that $K(r, \theta)$ is given by (20), we have

$$
\begin{aligned}
\int_{0}^{2 \pi} \sin \theta K(r, \theta) d \theta= & \sum_{i=0}^{n} \widetilde{a}_{i} r^{i} \int_{0}^{2 \pi} \cos ^{i} \theta \sin \theta d \theta \\
& +\sum_{i=0}^{m} \widetilde{b}_{i} r^{i+1} \int_{0}^{2 \pi} \cos ^{i} \theta \sin ^{2} \theta d \theta \\
& +\sum_{i=0}^{m} \widetilde{b}_{i} r^{i+3} \int_{0}^{2 \pi} \cos ^{i} \theta \sin ^{4} \theta d \theta \\
& +\sum_{i=0}^{l} \widetilde{c}_{i} r^{i+2} \int_{0}^{2 \pi} \cos ^{i} \theta \sin ^{3} \theta d \theta
\end{aligned}
$$

Hence

$$
\int_{0}^{2 \pi} \sin \theta K(r, \theta) d \theta=\sum_{\substack{i=0 \\ i \text { even }}}^{\mathrm{ev}(m)} \widetilde{b}_{i} r^{i+1} I_{i, 2}+\sum_{\substack{i=0 \\ i \text { even }}}^{\mathrm{ev}(m)} \widetilde{b}_{i} r^{i+3} I_{i, 4}
$$

Now noting that $P(r, \theta)$ is given by (11), we compute

$$
\begin{aligned}
\int_{0}^{2 \pi} & \frac{\sin \theta \cos \theta}{r} P^{2}(r, \theta) d \theta \\
= & \int_{0}^{2 \pi} \frac{\sin \theta \cos \theta}{r}\left(\sum_{i=0}^{n} a_{i} r^{i} \cos ^{i} \theta\right)^{2} d \theta \\
& +\int_{0}^{2 \pi} \frac{\sin \theta \cos \theta}{r}\left(\sum_{i=1}^{\operatorname{od}(m)} b_{i} r^{i+1} \cos ^{i} \theta \sin \theta\right)^{2} d \theta \\
& +\int_{0}^{2 \pi} \frac{\sin \theta \cos \theta}{r}\left(\sum_{i=0}^{l} c_{i} r^{i+2} \cos ^{i} \theta \sin ^{2} \theta\right)^{2} d \theta \\
& +\int_{0}^{2 \pi} \frac{\sin \theta \cos \theta}{r}\left(\sum_{i=1}^{\operatorname{od}(m)} b_{i} r^{i+3} \cos ^{i} \theta \sin ^{3} \theta\right)^{2} d \theta
\end{aligned}
$$

$$
\begin{array}{r}
+2 \int_{0}^{2 \pi} \frac{\sin \theta \cos \theta}{r}\left(\sum_{i=0}^{n} a_{i} r^{i} \cos ^{i} \theta\right) \\
\cdot\left(\sum_{\substack{j=1 \\
j \text { odd }}}^{\text {od }(m)} b_{j} r^{j+1} \cos ^{j} \theta \sin \theta\right) d \theta \\
+2 \int_{0}^{2 \pi} \frac{\sin \theta \cos \theta}{r}\left(\sum_{i=0}^{n} a_{i} r^{i} \cos ^{i} \theta\right)
\end{array}
$$$$
\cdot\left(\sum_{\substack{j=1 \\ j \text { odd }}}^{\text {od }(m)} b_{j} r^{j+3} \cos ^{j} \theta \sin ^{3} \theta\right) d \theta
$$$$
+2 \int_{0}^{2 \pi} \frac{\sin \theta \cos \theta}{r}\left(\sum_{i=0}^{n} a_{i} r^{i} \cos ^{i} \theta\right)
$$$$
\cdot\left(\sum_{j=0}^{l} c_{j} r^{j+2} \cos ^{j} \theta \sin ^{2} \theta\right) d \theta
$$$$
+2 \int_{0}^{2 \pi} \frac{\sin \theta \cos \theta}{r}\left(\sum_{\substack{i=1 \\ i \text { odd }}}^{\operatorname{od}(m)} b_{i} r^{i+1} \cos ^{i} \theta \sin \theta\right)
$$$$
\left(\sum_{\substack{j=1 \\ j \text { odd }}}^{\text {od }(m)} b_{j} r^{j+3} \cos ^{j} \theta \sin ^{3} \theta\right) d \theta
$$$$
+2 \int_{0}^{2 \pi} \frac{\sin \theta \cos \theta}{r}\left(\sum_{\substack{i=1 \\ i \text { odd }}}^{\operatorname{od}(m)} b_{i} r^{i+1} \cos ^{i} \theta \sin \theta\right)
$$$$
\cdot\left(\sum_{j=0}^{l} c_{j} r^{j+2} \cos ^{j} \theta \sin ^{2} \theta\right) d \theta
$$

$$
\begin{gathered}
+2 \int_{0}^{2 \pi} \frac{\sin \theta \cos \theta}{r}\left(\sum_{\substack{i=1 \\
i \text { odd }}}^{\text {od }(m)} b_{i} r^{i+3} \cos ^{i} \theta \sin ^{3} \theta\right) \\
\cdot\left(\sum_{j=0}^{l} c_{j} r^{j+2} \cos ^{j} \theta \sin ^{2} \theta\right) d \theta
\end{gathered}
$$

Hence

$$
\begin{aligned}
\int_{0}^{2 \pi} & \frac{\sin \theta \cos \theta}{r} P^{2}(r, \theta) d \theta \\
= & 2 \sum_{\substack{0 \leq i \leq \mathrm{ev}(n) \\
1 \leq j \leq \mathrm{od}(m) \\
i \text { even and } j \text { odd }}} a_{i} b_{j} r^{i+j} I_{i+j+1,2}
\end{aligned}
$$




$$
\begin{aligned}
& +2 \sum_{\substack{0 \leq i \leq \operatorname{ev}(n) \\
1 \leq j \leq \operatorname{od}(m) \\
i \text { even and } j \text { odd }}} a_{i} b_{j} r^{i+j+2} I_{i+j+1,4} \\
& +2 \sum_{\substack{1 \leq i \leq \operatorname{od}(m) \\
0 \leq j \leq \operatorname{ev}(l) \\
i \text { odd and } j \text { even }}} b_{i} c_{j} r^{i+j+2} I_{i+j+1,4} \\
& +2 \sum_{\substack{1 \leq i \leq \operatorname{od}(m) \\
0 \leq j \leq \operatorname{ev}(l) \\
i \text { odd and } j \text { even }}} b_{i} c_{j} r^{i+j+4} I_{i+j+1,6} \cdot
\end{aligned}
$$

From (55) and (57) we obtain

$$
\begin{aligned}
\int_{0}^{2 \pi} F_{2}(r, \theta) d \theta= & \sum_{\substack{i=0 \\
i \text { even }}}^{\text {ev }(m)} \widetilde{b}_{i} r^{i+1} I_{i, 2}+\sum_{\substack{i=0 \\
i \text { even }}} \widetilde{b}_{i} r^{i+3} I_{i, 4} \\
& -2 \sum_{\substack{0 \leq i \leq \mathrm{ev}(n) \\
1 \leq j \leq \mathrm{od}(m) \\
i \text { even and } j \text { odd }}} a_{i} b_{j} r^{i+j} I_{i+j+1,2} \\
& -2 \sum_{\substack{0 \leq i \leq \mathrm{ev}(n) \\
1 \leq j \leq \mathrm{od}(m) \\
i \text { even and } j \text { odd }}} a_{i} b_{j} r^{i+j+2} I_{i+j+1,4} \\
& -2 \sum_{\substack{1 \leq i \leq \mathrm{od}(m) \\
0 \leq j \leq \mathrm{ev}(l) \\
i \text { odd and } j \text { even }}} b_{i} c_{j} r^{i+j+2} I_{i+j+1,4} \\
& -2 \sum_{\substack{1 \leq i \leq \mathrm{od}(m) \\
0 \leq j \leq \mathrm{ev}(l) \\
i \text { odd and } j \text { even }}} b_{i} c_{j} r^{i+j+4} I_{i+j+1,6} .
\end{aligned}
$$

Then $F_{20}$ is the polynomial

$$
\begin{aligned}
& F_{20}(r)=\frac{1}{2 \pi}\left(\sum_{\substack{i=2 \\
i \text { even }}}^{\operatorname{ev}(n)} \sum_{\substack{j=1 \\
j \text { odd }}}^{\operatorname{od}(m)} i a_{i} b_{j} r^{i+j} A_{i j}\right. \\
& +\sum_{\substack{i=2 \\
i \text { even }}}^{\mathrm{ev}(n)} \sum_{\substack{j=1 \\
j \text { odd }}}^{\mathrm{od}(m)} i a_{i} b_{j} r^{i+j+2} B_{i j} \\
& -\sum_{\substack{1 \leq i \leq \mathrm{od}(m) \\
0 \leq j \leq \mathrm{ev}(n) \\
i \text { odd and } j \text { even }}} \frac{i+1}{j+1} a_{j} b_{i} r^{i+j} I_{i+j+1,2} \\
& +\sum_{\substack{i=1 \\
i \text { odd }}}^{\operatorname{od}(m)} \sum_{\substack{j=0 \\
j \text { even }}}^{\text {ev }(l)}(i+1) b_{i} c_{j} r^{i+j+2} D_{i j}
\end{aligned}
$$

$$
\begin{aligned}
& +\sum_{\substack{i=0 \\
i \text { even }}}^{\operatorname{ev}(l)} \sum_{\substack{j=1 \\
j \text { odd }}}^{\operatorname{od}(m)}(i+2) c_{i} b_{j} r^{i+j+2} E_{i j} \\
& +\sum_{\substack{i=0 \\
i \text { even }}}^{\operatorname{ev}(l)} \sum_{\substack{j=1 \\
j \text { odd }}}^{\text {od }(m)}(i+2) c_{i} b_{j} r^{i+j+4} F_{i j} \\
& -\sum_{\substack{1 \leq i \leq \mathrm{od}(m) \\
0 \leq j \leq \mathrm{ev}(n) \\
i \text { odd and } j \text { even }}} \frac{i+3}{j+1} a_{j} b_{i} r^{i+j+2} I_{i+j+1,4} \\
& +\sum_{\substack{i=1 \\
i \text { odd }}}^{\operatorname{od}(m)} \sum_{\substack{j=0 \\
j \text { even }}}^{\operatorname{ev}(l)}(i+1) b_{i} c_{j} r^{i+j+4} H_{i j} \\
& +\sum_{\substack{i=0 \\
i \text { even }}}^{\mathrm{ev}(m)} \widetilde{b}_{i} r^{i+1} I_{i, 2}+\sum_{\substack{i=0 \\
i \text { even }}}^{\mathrm{ev}(m)} \widetilde{b}_{i} r^{i+3} I_{i, 4} \\
& -2 \sum_{\substack{0 \leq i \leq \operatorname{ev}(n) \\
1 \leq j \leq \operatorname{od}(m) \\
i \text { even and } j \text { odd }}} a_{i} b_{j} r^{i+j} I_{i+j+1,2} \\
& -2 \sum_{\substack{0 \leq i \leq \operatorname{ev}(n) \\
1 \leq j \leq \operatorname{od}(m) \\
i \text { even and } j \text { odd }}} a_{i} b_{j} r^{i+j+2} I_{i+j+1,4} \\
& -2 \sum_{\substack{1 \leq i \leq \mathrm{od}(m) \\
0 \leq j \leq \operatorname{ev}(l) \\
i \text { odd and } j \text { even }}} b_{i} c_{j} r^{i+j+2} I_{i+j+1,4} \\
& \left.-2 \sum_{\substack{1 \leq i \leq \mathrm{od}(m) \\
0 \leq j \leq \mathrm{ev}(l) \\
i \text { odd and } j \text { even }}} b_{i} c_{j} r^{i+j+4} I_{i+j+1,6}\right) .
\end{aligned}
$$

Note that in order to find the positive roots of $F_{20}$ after dividing by $r$, we must find the zeros of a polynomial in the variable $r^{2}$ of degree equal to the $\max \{((\operatorname{ev}(n)+\operatorname{od}(m)+2)-$ $1) / 2,((\mathrm{ev}(l)+\operatorname{od}(m)+4)-1) / 2,((\mathrm{ev}(m)+3)-1) / 2\}$, we conclude that $F_{20}$ has at most $\max \{[n / 2]+[(m+1) / 2],[l / 2]+$ $[(m+3) / 2],[(m+2) / 2]\}$ positive roots. Hence $(\mathrm{b})$ of Theorem 1 is proved.

\section{Conflict of Interests}

The authors declare that there is no conflict of interests regarding the publication of this paper.

\section{References}

[1] D. Hilbert, "Mathematische problems, lecture in Second International Congress of Mathematics, Paris, 1900," Nachrichten von 
der Gesellschaft der Wissenschaften zu Göttingen. MathematischPhysikalische Klasse, vol. 5, pp. 253-297, 1900, English translation Bulletin of the American Mathematical Society, vol. 8, pp. 437-479, 1902.

[2] I. S. Kukles, "Sur quelques cas de distinction entre un foyer et un centre," Comptes Rendus de l'Académie des Sciences de l'URSS (N. S.), vol. 42, pp. 208-211, 1944 (French).

[3] C. Rousseau, D. Schlomiuk, and P. Thibaudeau, "The centres in the reduced Kukles system," Nonlinearity, vol. 8, no. 4, pp. 541$569,1995$.

[4] C. Rousseau and B. Toni, "Local bifurcations of critical periods in the reduced Kukles system," Canadian Journal of Mathematics, vol. 49, no. 2, pp. 338-358, 1997.

[5] C. J. Christopher and N. G. Lloyd, "On the paper of X. Jin and D. Wang concerning the conditions for a centre in certain cubic systems," Bulletin London Mathematical Society, vol. 22, pp. 512, 1990.

[6] H. Zang, T. Zhang, Y.-C. Tian, and M. O. Tadé, "Limit cycles for the Kukles system," Journal of Dynamical and Control Systems, vol. 14, no. 2, pp. 283-298, 2008.

[7] A. P. Sadovskii, "Cubic systems of nonlinear oscillations with seven limit cycles," Differential Equations, vol. 39, no. 4, pp. 505516, 2003.

[8] J. Chavarriga, E. Sáez, I. Szántó, and M. Grau, “Coexistence of limit cycles and invariant algebraic curves for a Kukles system," Nonlinear Analysis. Theory, Methods \& Applications, vol. 59, no. 5, pp. 673-693, 2004.

[9] A. Boulfoul and A. Makhlouf, "Limit cycles of the generalized polynomial Liénard differential equation," Annals of Differential Equations, vol. 28, no. 2, pp. 127-131, 2012.

[10] J. Llibre and A. C. Mereu, "Limit cycles for generalized Kukles polynomial differential systems," Nonlinear Analysis. Theory, Methods \& Applications, vol. 74, no. 4, pp. 1261-1271, 2011.

[11] A. Buica and J. Llibre, "Averaging methods for Önding periodic orbits vai Brouwer degree," Bulletin des Sciences Mathématiques, vol. 128, pp. 7-22, 2004.

[12] J. A. Sanders and F. Verhulst, Averaging Methods in Nonlinear Dynamical Systems, vol. 59 of Applied Mathematical Sciences, Springer, New York, NY, USA, 1985.

[13] F. Verhulst, Nonlinear Differential Equations and Dynamical Systems, Springer, Berlin, Germany, 1996. 


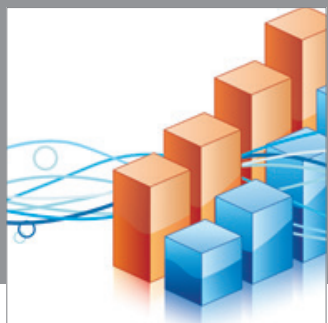

Advances in

Operations Research

mansans

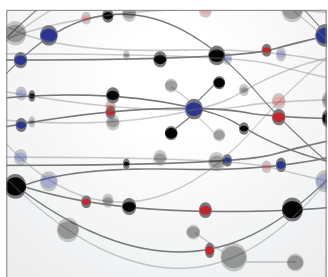

The Scientific World Journal
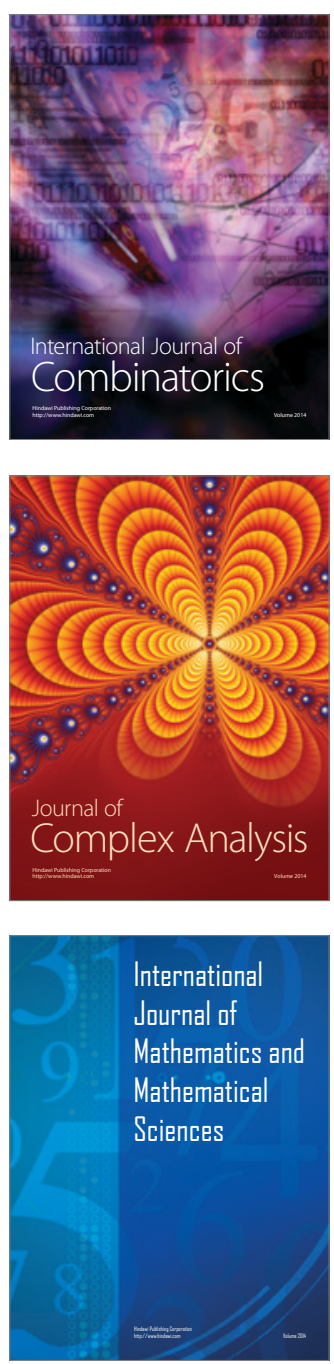
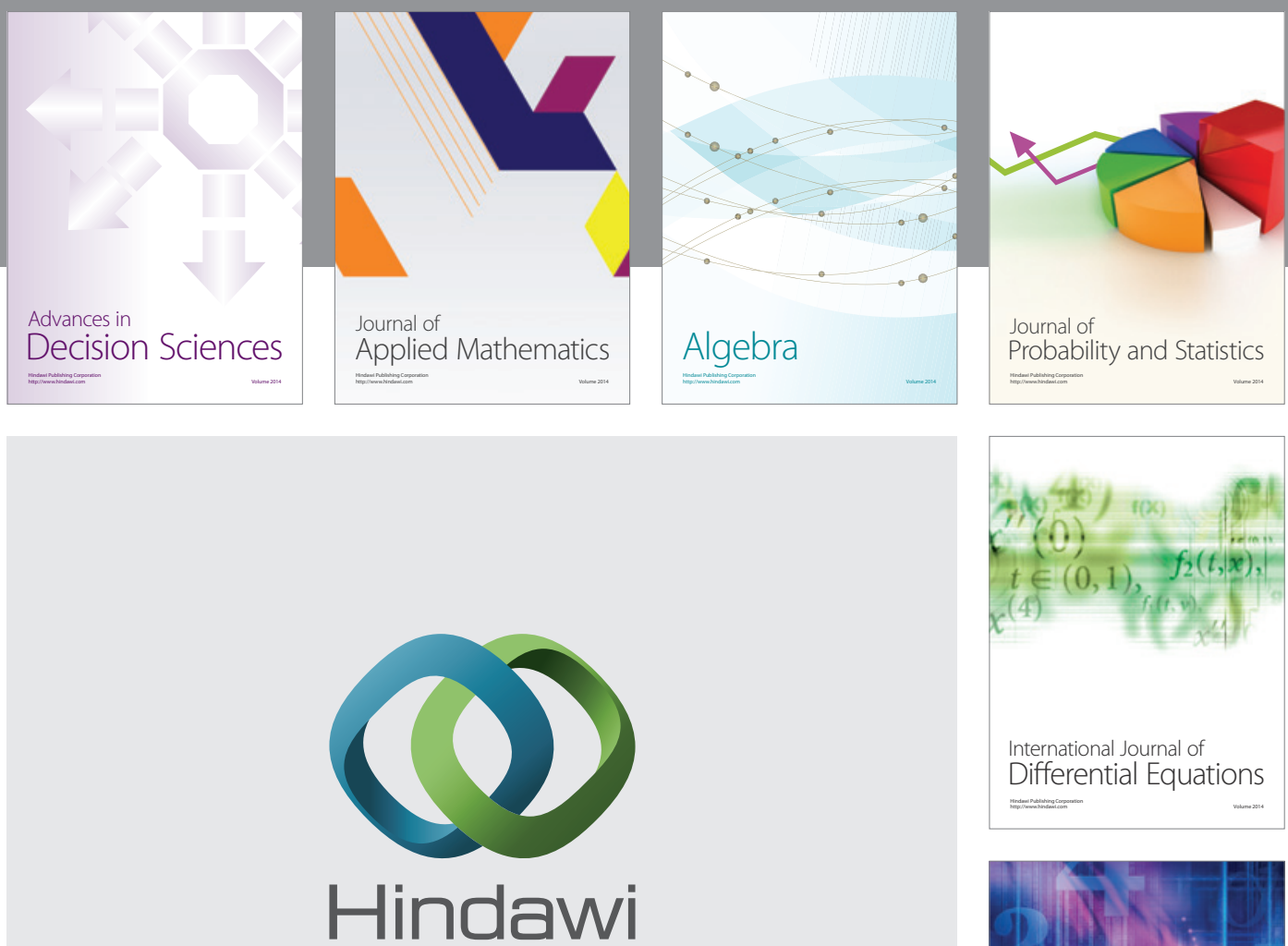

Submit your manuscripts at http://www.hindawi.com
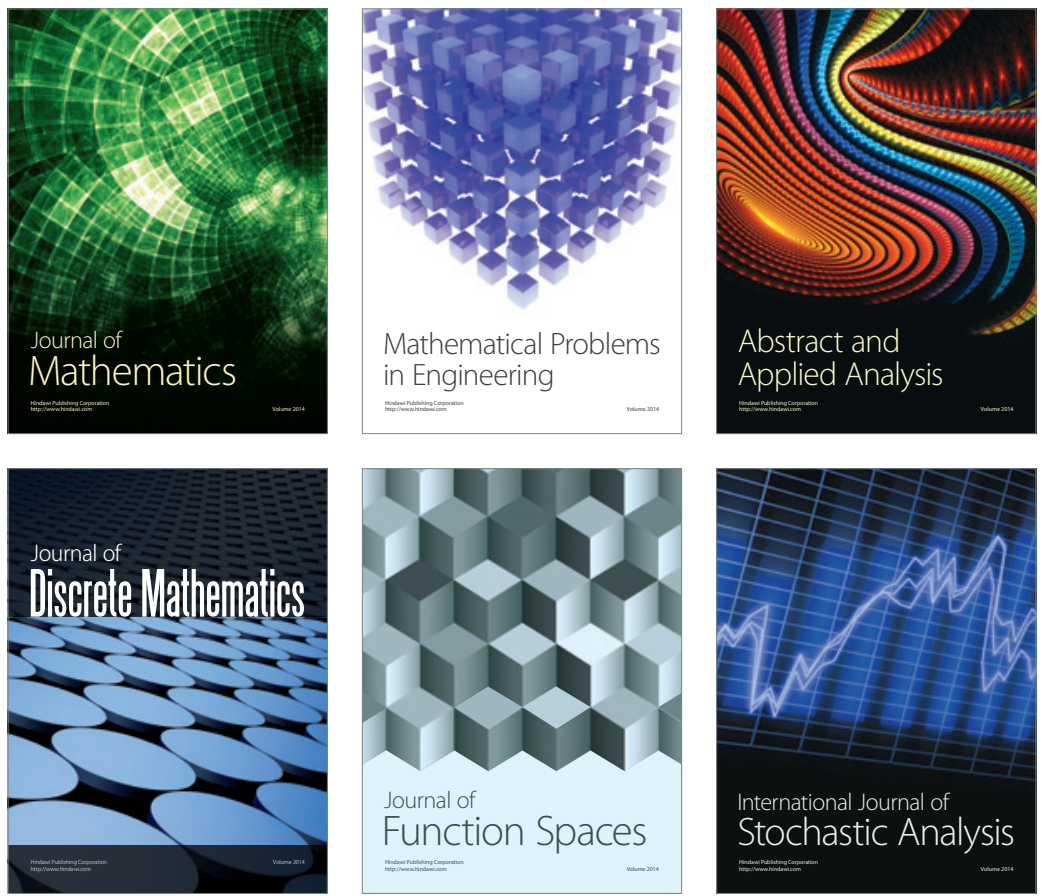

Journal of

Function Spaces

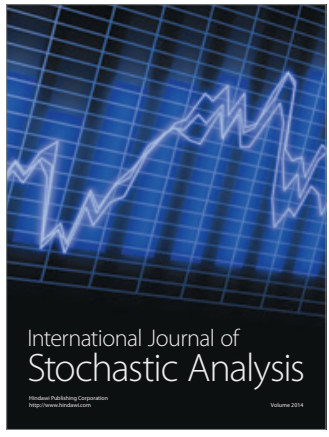

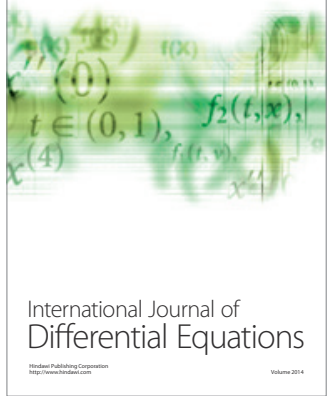
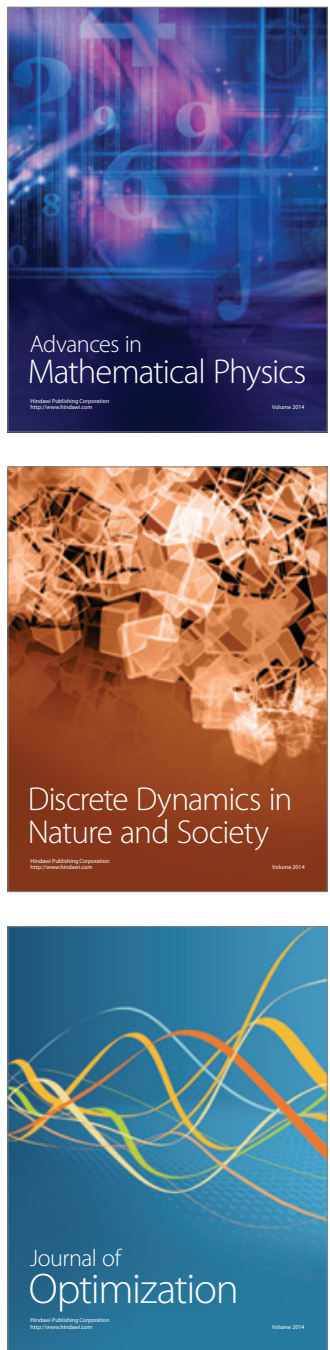